\title{
Deciphering Optimal Strategy of Microbial Fuel Cell-Assisted Natural Bioremediation of Textile Dyes
}

\author{
Bor-Yann Chen ${ }^{*}$, Pei-Lin Yueh ${ }^{1}, K_{e}$ Han $^{2}$, Lian-Jie Qin² \\ ${ }^{1}$ Department of Chemical and Materials Engineering, National I-Lan University, I-Lan 26047, Taiwan \\ bychen@niu.edu.tw; boryannchen@yahoo.com.tw; j93070534@yahoo.com.tw \\ ${ }^{2}$ School of Environmental and Materials Engineering, Yan-Tai University, 264005, China \\ hanke1514@163.com; lianjieqin@126.com
}

\begin{abstract}
Prior study provided a novel evaluation scheme to quantitatively reveal "synergistic" stimulation of microbial fuel cell (MFC)-assisted dye decolorization for industrial practicability. This follow-up work tended to optimize electron transfer (ET) characteristics for maximal performance of dye decolorization via simultaneous bioelectricity generation and dye decolorization (SBG\&DD) in MFCs. Supplementation of decolorized metabolites of textile dyes apparently facilitated both rates of BG and DD. However, higher dye concentration seemed to be more favourable to electron distribution dominated for colour removal. Moreover, whether DM-supplementation was synergistic or antagonistic to DD and BG strongly depended upon interactive characteristics of DMs and mother dye species with salts for practical operation. This plausible analysis suggested that the most desirable mode of MFC-aided pollutant degradation should be batch or plug flow reactor modes of operation at low conversions for optimal bioremediation.
\end{abstract}

Keywords: Microbial fuel cell, Bioelectricity generation, Reductive decolorization, Natural bioremediation

\section{Introduction}

In-situ and on site bioremediation to environmental problems is usually considered the most appropriate treatment for pollutant degradation with concerns of environmental friendliness and cost-effectiveness due to applications of indigenous microbes for sustainable development [1]. Recently, microbial fuel cells (MFCs) were rapidly evolving methods to effectively accelerate decomposition of pollutants with simultaneous energy recycling through organics oxidation [2]. In particular, MFCs could catalytically implement simultaneous contaminant degradation and bioelectricity generation for natural attenuation [3][4]. Among biodegradation of various organics, biodecolorization of azo dye(s) is carried out via cometabolism by extracting energy from oxidation of primary source (e.g., organic substrate). To promote electrontransferring capability of MFCs for wastewater (WW) treatment, one of the most intriguing alternatives prevailing recently was exogenous supplementation of electron shuttles (ESs) below toxicity thresholds [5]. Recently, literature [4][6] revealed that decolorized metabolites (DMs) of textile dyes might function as ESs in MFCs to enhance the performance of electron transfer (ET)-driven colour removal and power generation. In addition, to enhance pollutant decontamination, decolorized intermediates containing functional substituents of $-\mathrm{OH},-\mathrm{NH}_{2},-\mathrm{N}\left(\mathrm{CH}_{3}\right) \mathrm{H}$ and $-\mathrm{N}\left(\mathrm{CH}_{3}\right)_{2}$ were found to act as ESs [7] for dye decolorization. Regarding MFC-based pollutant degradation, Luo et al. [8] revealed that phenol degradation rate of MFC at closed-circuit (CC) operation (ca. $\left.4.2 \mathrm{mg} \mathrm{L}^{-1} \mathrm{~h}^{-1}\right)$ was apparently higher than that of open-circuit (OC) operation $\left(3.3 \mathrm{mg} \mathrm{L}^{-1}\right.$ $\mathrm{h}^{-1}$ ). As Sun et al. [9] also disclosed, compared to OC conditions (i.e., external resistance $\mathrm{R}_{\mathrm{ex}}=\infty$ ), color removal performance of $\mathrm{R}_{\mathrm{ex}}=5000 \Omega, 500 \Omega, 50 \Omega$ at $24 \mathrm{~h}$ increased $1.24,1.51$ and 1.64 fold due to MFC-aided stimulation of electron transfer (ET) for dye decolorization. In addition, Gajda et al. [10] compared the performance of OC and CC MFCs, revealing that the current generation of CC was ca. 3 fold of OC. However, systematic and quantitative analyses upon MFC-based pollutant degradation are still remained open to be explored. Here, this study quantitatively revealed not only the promising feasibility of using MFCs for pollutant decontamination, but also optimal performance of MFC-aided bioremediation. In fact, Han et al. [11] quantitatively revealed that accumulation of DMs in MFCs could auto-catalytically enhance ca. 40-70\% wastewater decolorization for industrial practicability. As textile effluents usually contained high concentrations of salts and auxiliary agents affecting treatment efficiency, this study also compared operation performance of MFC-aided dye decolorization at different $\mathrm{NaCl}$ concentrations. As indicated herein, whether DM-supplementation was synergistic or antagonistic to decolorization strongly depended upon interactive characteristics of DM and mother dye species with salts. This study also 
suggested that non-flow reactor modes of operation with recycled DM to enhance ET-efficiency were the most appropriate contacting patterns of organics decomposition for optimal MFC-based bioremediation.

\section{Materials and Methods}

\subsection{Chemicals, bacterial strains and culture conditions}

Textile dyes- reactive green 19 (RG19), reactive blue160 (RBu160), reactive red 198 (RR198), Orange I and malachite green (MG) (Everlight Chemical Ltd., Taipei, Taiwan) were used to colour removal. Inoculated bacterium Shewanella sp. WLP72 was isolated from gut microbial consortia of deep seawater-fed Babylonia areolata which was harvested from Hualien, Taiwan. Bacteria were cultured in sterilized Luria-Bertani (LB) broth medium. Decolorized metabolites (DMs) of textile dyes were obtained as described in Han et al. [11].

\subsection{Experimental operations}

Single-chamber WLP72-seeded MFCs were constructed as described elsewhere [4]. For bacterial acclimatization in MFCs, nutrient augmentation was conducted every 2 days to ensure no other factors limiting electrochemical activity of cells during operation. Regarding SBG\&DD in Shewanella sp. WLP72-inoculated MFCs, impulse injections of RBu160 or DMs at designated concentrations were serially added into MFCs. After 2 days testing, to remove residual chemicals, $50 \%$ of the cell broth was replaced by fresh RBu160-bearing medium for next cycle of serial MFC inspections. The voltage difference between the anode and cathode of MFCs and dye concentrations were continuously recorded [12]. Batch-fed MFCs with impulse injections of test metabolites (e.g., DM of RG19, RBu160 and MG) were conducted serially for comparative analysis.

\subsection{Electrochemical measurements and analysis}

For evaluation of power generation, cell voltage was automatically measured (set at one data point per minute) using a data acquisition system (DAS 5020; Jiehan Technology Corp.) through external resistance $\mathrm{R}_{\text {out }}=1 \mathrm{~K} \Omega$. A relatively high resistance (1000 ohms) was intentionally used to compare with prior results [12].

\subsection{Kinetic model analysis}

Regarding initial rate inspections, initial current generation from oxidation of energy sources (e.g., organic matters) in anodic biofilm could be formulated in a first order decay rate equation [11].

$$
\frac{d I}{d t}=-k I \quad I\left(t_{0}\right)=I_{0}
$$

where I, $\mathrm{t}$ and $\mathrm{k}$ were electric current through the resistance, time and decay rate constant, respectively. According to ohm's law (i.e., $\mathrm{V}=\mathrm{I} \times \mathrm{R}$ ), one could reformulate the differential equation as

$$
\frac{d V}{d t}=-k V . \quad V\left(t_{0}\right)=V_{0}
$$

The analytical solution is $V=V_{0} \exp \left(-k\left(t-t_{0}\right)\right)$.

If the initial rate of ET could be termed as

$$
\frac{d e^{-}}{d t_{t=t_{0}}} \propto \frac{d I}{d t_{t=t_{0}}}=\frac{1}{R} \frac{d V}{d t}{ }_{t=t_{0}}=-\frac{1}{R} k V_{0} \exp \left(-k\left(t-t_{0}\right)\right)_{t=t_{0}}=-\frac{1}{R} k V_{0}
$$

and initial decolorization rate was determined as $-\frac{d[D y e]}{d t}=I D R$, then the ratio of rate of electrons transferred of 
$\mathrm{IDR}_{\mathrm{d}}$ to $\mathrm{k}_{\mathrm{e}} \mathrm{V}_{0 \mathrm{e}}$ could be defined as $-\frac{d[D y e]}{d e^{-}} \propto \frac{I D R_{d}}{k_{e} V_{0 e}}=a$. Higher $a$ values simply suggested that ET was more favourable for dye decolorization than bioelectricity generation. Overall "stoichiometric" ratio of ET with respect to IDR at DD alone (i.e., $\operatorname{IDR}_{d}$ ) was defined as $\phi=\eta / \mathrm{IDR}_{\mathrm{d}}$.

\section{Results and Discussion}

\subsection{Quantitative assessment upon SBG\&DD}

Prior study [11] indicated that supplementation of DMs of textile dyes might significantly augment efficiencies of dye decolorization. In addition, MFC-aided bioremediation would be the promising method of dye decontamination due to synergistic stimulation of ET-driven MFC operation. Here, to unravel how dye decolorization could be synergistically enhanced in MFCs-assisted operation, comparison upon SBG\&DD, only bioelectricity-generation (BG alone) and dye decolorization alone (DD alone) were carried out in MFC reactors (Table 1 and Fig. 1(a),1(b); [11]). The rankings of initial decolorization rate (IDR) and initial decay rate of BG (IRBG) $k V_{0}$, in decreasing order, could be found as follows (Table 1 and Fig. 1(a); [11]): (1) IDR (unit: $\mathrm{mg} \mathrm{L}^{-1} \mathrm{~h}^{-1}$ ): RG19-DM (75.6) > RBu160-DM (66.8) > MG-DM (64.3) > MB (53.3) Blank (51.4), (2) IRBG $k V_{0}$ (unit: $\mathrm{mV} \mathrm{h}^{-1}$ ): RBu160-DM (5.976) $\cong \mathrm{MG-DM} \mathrm{(5.725)} \mathrm{\cong} \mathrm{RG19-DM} \mathrm{(5.642)} \mathrm{>} \mathrm{MB} \mathrm{(5.021)}$ Blank (4.950). Apparently, the most promising DM to enhance DD was RG19-DM $\left(\bar{a}=13.4 \mathrm{mg} \mathrm{L}^{-1} \mathrm{mV}^{-1}, \eta=128.52 \mathrm{mg} \mathrm{L}^{-}\right.$ ${ }^{1} \mathrm{~h}^{-1}$ ) as the maximal percentages of ET could be contributed to DD.

Evidently, augmentation of DMs synergistically stimulated both performance of DD and BG. This is consistent with prior finding of synergistic stimulation via ET-directed SBG\&DD in MFCs [11]. In addition, the ranking of overall "stoichiometric" ratio of electron-transport $\phi$ due to MFC-assisted SBG\&DD could be obtained as follows: Blank (1.699) MB (1.698) > MG-DM (1.572) > RBu160-DM (1.456) > RG19-DM (1.413) (Fig. 1(a),1(b),1(c); Table 1). Although higher values of $\phi$ were shown in DM-absent MFC operations, overall equivalent ET $(\eta)$ capabilities were still lower than DMpresent cases. As all of the ratios were apparently great than unity (ca. $1.413 \sim 1.699$ ), using MFC as the method for pollutant degradation would thus be electrochemically favourable through synergistic stimulation of ET.

To consider practicability for treatment of high level salts-bearing textile dye effluents, this study also compared operation efficiency of MFC-assisted decolorization. As indicated in Table 2, without DM-supplementation, 20, $30 \mathrm{~g} \mathrm{~L}^{-1}$ $\mathrm{NaCl}$ would resist ET capabilities of MFC ( $\phi=0.61-0.62)$ due to salt stress to WLP72. The presence of DM could possibly overcome such stress to effectively express for ET capabilities to BG and DD. However, whether DM supplementation showed synergistic (e.g., Orange I-DM) or antagonistic (e.g., RR198-DM) interactions with NaCl to DD and BG might strongly depend upon characteristics of DM and mother dyes to receptor WLP72.

Table 1: Comparative list of performance of SBG\&DD, BG and DD alone of MFCs using different species of DMs ${ }^{\perp \dagger}$.

\begin{tabular}{|l|l|l|l|l|c|c|l|l|}
\hline $\begin{array}{l}\text { Metabolite } \\
\text { species }\end{array}$ & IDR & $k V_{0}$ & $\mathrm{IDR}_{\mathrm{d}}$ & $k_{\mathrm{e}} V_{0 \mathrm{e}}$ & $a=\frac{\mathrm{IDR}_{\mathrm{d}}}{\mathrm{k}_{\mathrm{e}} \mathrm{V}_{0 \mathrm{e}}}$ & $\bar{a}=\frac{\mathrm{IDR}}{\mathrm{kV}_{0}}$ & $\eta=\left(k V_{0}\right) a+I D R$ & $\phi=\frac{\eta}{I D R_{\mathrm{d}}}$ \\
\hline Blank & 51.4 & 5.021 & 60.89 & 5.871 & 10.37 & 10.2 & 103.47 & 1.699 \\
\hline MB & 53.3 & 4.950 & 60.04 & 6.108 & 9.830 & 10.8 & 101.96 & 1.698 \\
\hline RG19-DM & 75.6 & 5.642 & 90.95 & 9.696 & 9.380 & 13.4 & 128.52 & 1.413 \\
\hline RBu160-DM & 66.8 & 5.746 & 81.28 & 9.058 & 8.973 & 11.6 & 118.36 & 1.456 \\
\hline MG-DM & 64.3 & 5.725 & 77.54 & 7.704 & 10.06 & 11.2 & 121.89 & 1.572 \\
\hline
\end{tabular}

${ }^{\perp}$ Subscripts $\mathrm{d}$ and e denoted $\mathrm{DD}$ and $\mathrm{BG}$ alone, respectively.

'Parameters IDR $\left(\mathrm{mg} \mathrm{L}^{-1} \mathrm{~h}^{-1}\right)$, Maximal output voltage $V_{0}(\mathrm{mV})$, Decay rate constant $k\left(\mathrm{~h}^{-1}\right)$, The ratio of electrons transferred of $I D R_{\mathrm{d}}$ to $k_{e} V_{0_{e}} ; a=\frac{I D R_{d}}{k_{e} V_{0_{e}}}\left(\mathrm{mg} \mathrm{L}^{-1} \mathrm{mV}^{-1}\right)$, The ratio of electrons transferred of $I D R$ to $\mathrm{kV}_{0} ; \bar{a}=\frac{I D R}{\mathrm{kV}_{0}}\left(\mathrm{mg} \mathrm{L}^{-1} \mathrm{mV}^{-1}\right)$, Overall equivalent electrons transferred $\eta=I D R+a\left(k V_{0}\right)\left(\mathrm{mg} \mathrm{L}^{-1} \mathrm{~h}^{-1}\right)$, Overall "stoichiometric" ratio of electron transport $\phi=\eta / I D R_{\mathrm{d}}(-)$ were defined in [11].

Table 2: Comparison of overall "stoichiometric" ratio of ET $\phi$ of MFCs using different species of DM at different NaCl concentrations. 


\begin{tabular}{|l|l|l|l|}
\hline$\phi$ value & $10 \mathrm{~g} \mathrm{~L}^{-1}$ & $20 \mathrm{~g} \mathrm{~L}^{-1}$ & $30 \mathrm{~g} \mathrm{~L}^{-1}$ \\
\hline Blank & 1.58 & 0.61 & 0.62 \\
\hline RR198-DM & 1.75 & 1.47 & 1.07 \\
\hline $\begin{array}{l}\text { Orange I- } \\
\text { DM }\end{array}$ & 1.15 & 1.34 & 1.47 \\
\hline
\end{tabular}

(a)

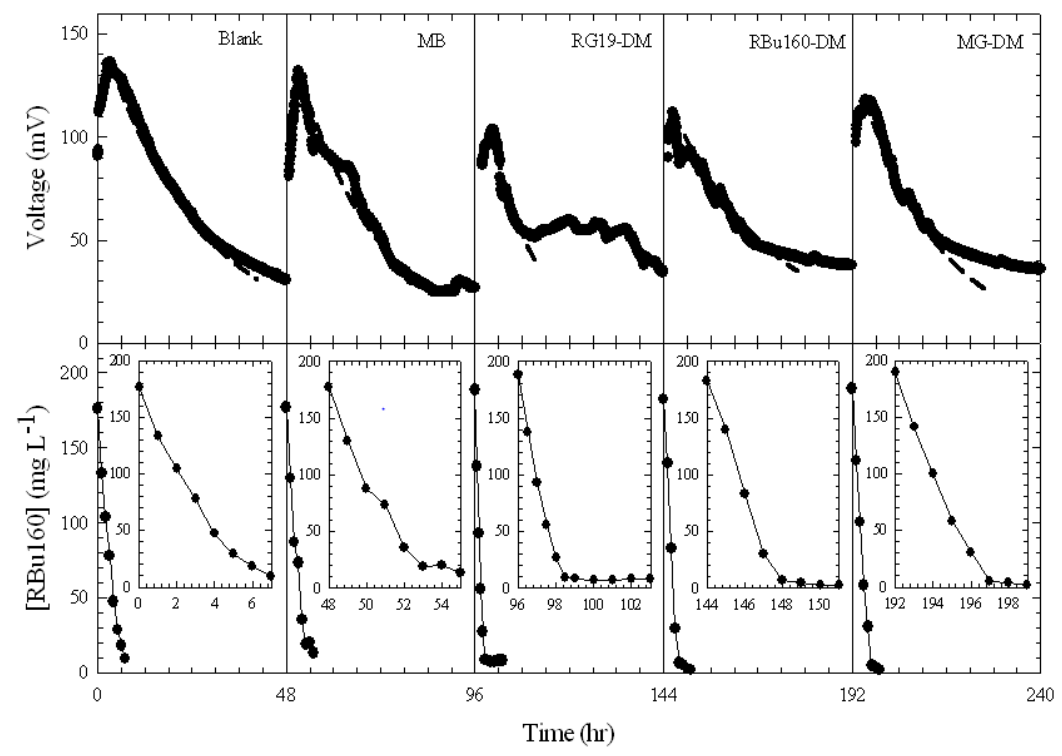

(b)

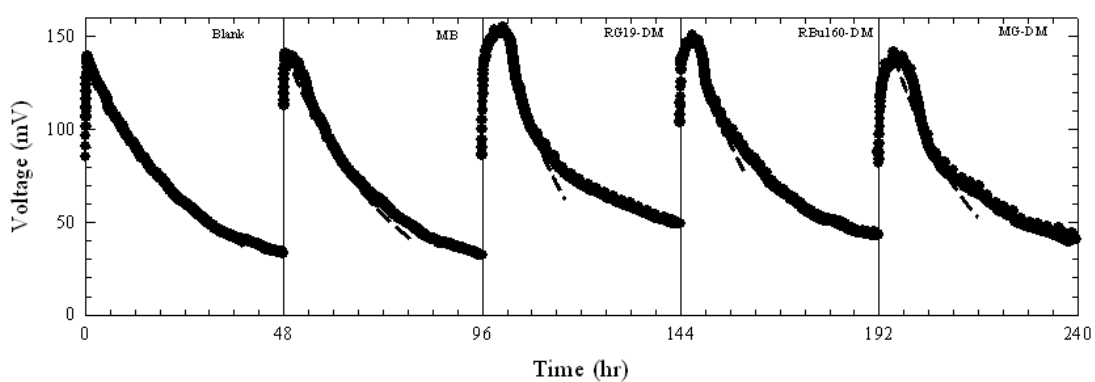

(c)

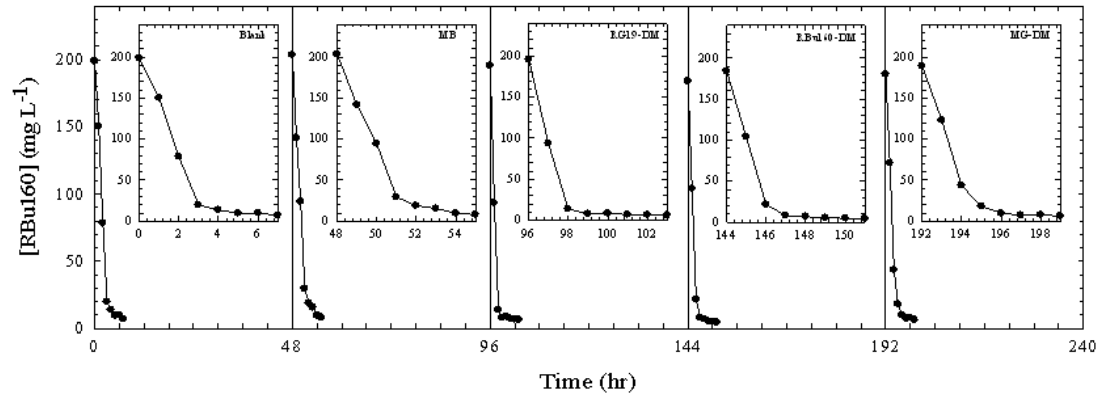

Fig. 1: Time courses of decolorization of RBu160 and power generation of (a) SBG\&DD, (b) BG alone and (c) DD alone of Shewanella sp. WLP72 (external resistance = $1 \mathrm{~K} \Omega$ ) (data modified from [11]).

\subsection{Optimal strategy of operation}


Dye concentration: As mentioned in [11], RG19 owned the most significant redox-mediating capability to simulate BG and DD compared to RBu160 and MG. To reveal optimal dye concentration of MFC-aided treatment, different loadings of RBu160 (i.e., 100, 200, 400, $600 \mathrm{mg} \mathrm{L}^{-1}$; Table 3) were supplemented in RG19-DM-bearing MFCs for SBG\&DD. The rankings of initial decolorization rate (IDR) in decreasing order, could be shown as follows (time course data not shown): (1) IDR (unit: $\left.\mathrm{mg} \mathrm{L}^{-1} \mathrm{~h}^{-1}\right): 184.3\left(600 \mathrm{mg} \mathrm{L}^{-1}\right)>83.7\left(400 \mathrm{mg} \mathrm{L}^{-1}\right)>58.4\left(200 \mathrm{mg} \mathrm{L}^{-1}\right)>50.3\left(100 \mathrm{mg} \mathrm{L}^{-1}\right)$, (2) IRBG (unit: $\left.\mathrm{mV} \mathrm{h}^{-1}\right): 4.020\left(100 \mathrm{mg} \mathrm{L}^{-1}\right)>3.579\left(200 \mathrm{mg} \mathrm{L}^{-1}\right)>2.763\left(400 \mathrm{mg} \mathrm{L}^{-1}\right)>2.563\left(600 \mathrm{mg} \mathrm{L}^{-1}\right)$. Regarding DD alone, the ranking of initial decolorization rate (IDR; $\mathrm{mg} \mathrm{L}^{-1} \mathrm{~h}^{-1}$ ), in decreasing order, could be listed as follows (Table 3): 203.4 (600 $\left.\mathrm{mg} \mathrm{L}{ }^{-1}\right)>97.6\left(400 \mathrm{mg} \mathrm{L}^{-1}\right)>67.4\left(200 \mathrm{mg} \mathrm{L}^{-1}\right)>65.3\left(100 \mathrm{mg} \mathrm{L}^{-1}\right)$.

Apparently, as concentration of RBu160 increased, decolorization performance (IDR) gradually increased, but power generation (IRBG) gradually decreased due to competition between BG and DD. By using of DM obtained from decolorization of $200 \mathrm{mg} \mathrm{L}^{-1} \mathrm{RG} 19$ and the value of $a=9.38 \mathrm{mg} \mathrm{L}^{-1} \mathrm{mV}^{-1}$ adopted [11], the ranking of overall stoichiometric ratio of electron transfer (ET) $\phi$ in MFCs were $1.36\left(200 \mathrm{mg} \mathrm{L}^{-1}\right)>1.35\left(100 \mathrm{mg} \mathrm{L}^{-1}\right)>1.12\left(400 \mathrm{mg} \mathrm{L}^{-1}\right)>1.02\left(600 \mathrm{mg} \mathrm{L}^{-}\right.$ ${ }^{1}$ ). That is, optimal RBu160 concentration for dye decontamination was ca. $200 \mathrm{mg} \mathrm{L}^{-1}$ for MFCs bearing with same level of DM of RG19. Evidently, higher levels of RBu160 would gradually suppress such ET efficiency. Moreover, the $\phi$ values approached to unity (basic level) at high RBu160 concentration very likely due to insufficient number of electrons available for $\mathrm{BG}$ or $\mathrm{DD}$.

Table 3: Comparative list of performance of SBG\&DD and DD alone of MFCs at different initial RBu160 concentrations ${ }^{\dagger}$.

\begin{tabular}{|l|l|l|l|l|l|c|}
\hline $\begin{array}{l}\mathrm{RBu} 160 \\
\left(\mathrm{mg} \mathrm{L}^{-1}\right)\end{array}$ & IDR & $k V_{0}$ & IDR & $\bar{a}=\frac{\mathrm{IDR}}{\mathrm{kV}_{0}}$ & $\eta=\left(k V_{0}\right) a+I D R$ & $\phi=\frac{\eta}{I D R_{\mathrm{d}}}$ \\
\hline 100 & 50.3 & 4.020 & 65.3 & 12.5 & 88.01 & 1.35 \\
\hline 200 & 58.4 & 3.579 & 67.4 & 16.3 & 91.97 & 1.36 \\
\hline 400 & 83.7 & 2.763 & 97.6 & 30.3 & 109.61 & 1.12 \\
\hline 600 & 184.3 & 2.563 & 203.4 & 71.9 & 208.34 & 1.02 \\
\hline
\end{tabular}

$\dagger$ RG19-DM was used as model ES.

Subscripts $\mathrm{d}$ and e denoted DD and BG alone, respectively.

Parameters and units of IDR, $V_{0}, k, \bar{a}, \eta$, $\phi$ were defined in Table 1 and $a=\frac{I D R_{d}}{k_{e} V_{0}}=9.38 \mathrm{mg} \mathrm{L}^{-1} \mathrm{mV}^{-1}$ was adopted from [11].

Decolorized metabolites: Although DMs could act as ESs, supplementation of DMs should still maintain within certain levels to minimize toxicity of ESs for maximal expression of BG and DD. With different dosages of RG19-DMs obtained from decolorization of RG19 at 200, 400, 600, $800 \mathrm{mg} \mathrm{L}^{-1}$, MFCs-assisted dye decolorization using RBu160 at 200 $\mathrm{mg} \mathrm{L}^{-1}$ was implemented (Table 4). The rankings of IDR and overall stoichiometric ratio of ET $\phi$ was ranked as 1.37 (600 $\left.\mathrm{mg} \mathrm{L}^{-1}\right)>1.36\left(400 \mathrm{mg} \mathrm{L}^{-1}\right)>1.31\left(200 \mathrm{mg} \mathrm{L}^{-1}\right)>1.28\left(800 \mathrm{mg} \mathrm{L}^{-1}\right)$. Compared to Tables 3 and 4, supplementation of DM below $600 \mathrm{mg} \mathrm{L}^{-1}$ would evidently increase BG performance. However, the ratios of ET of IDR to $k V_{0}$ were almost invariant (ca. 12.52-12.57; Table 3), indicating that such ET-stimulating phenomena seemed to augment BG and DD in parallel. Moreover, optimal concentration of DM for electron-shuttling was ca. $600 \mathrm{mg} \mathrm{L}^{-1}$. These all $\phi$ values were greater than unity, suggesting that MFC technology was of course very promising for textile dye-bioremediation. 0

System optimization: According to Levenspiel [13], if BG and DD were considered parallel reactions in ET-driven MFCs, the relative ratios of initial rates of DD and BG (i.e., $a$ and $\bar{a}$ in Tables) would gradually increase if [RBu160] increased (Table 2). That is, to maximize DD performance in MFCs, RBu160 concentration should be "as large as possible" (i.e., using a batch or plug flow reactor) to minimize reactor size for optimization. However, electron distribution seemed to invariant at different DMs (Table 3), suggesting that selectivity of ET was unaffected by the type of reactor selected. That is, non flow-types of reactor operation with DM-bearing recycles would be the most appropriate modes for MFC-aided textile dye decontamination. 
Table 4: Comparison upon performance of SBG\&DD and DD alone of MFCs bearing with DMs obtained from decolorization of RG19 at different concentrations ${ }^{\dagger}$.

\begin{tabular}{|l|l|l|l|l|l|c|}
\hline $\begin{array}{l}\text { RG19-DM } \\
\left(\mathrm{mg} \mathrm{L}^{-1}\right)\end{array}$ & IDR & $k V_{0}$ & $\mathrm{IDR}_{\mathrm{d}}$ & $\bar{a}=\frac{\mathrm{IDR}}{\mathrm{kV}_{0}}$ & $\eta=\left(k V_{0}\right) a+I D R$ & $\phi=\frac{\eta}{I D R_{\mathrm{d}}}$ \\
\hline 200 & 57.3 & 4.573 & 76.7 & 12.53 & 100.2 & 1.31 \\
\hline 400 & 62.3 & 4.976 & 80.2 & 12.52 & 109.0 & 1.36 \\
\hline 600 & 66.7 & 5.305 & 84.7 & 12.57 & 116.5 & 1.37 \\
\hline 800 & 58.9 & 4.694 & 80.4 & 12.55 & 102.9 & 1.28 \\
\hline
\end{tabular}

†Part of data not shown.

Subscripts d and e denoted DD and BG alone, respectively.

Parameters and units of IDR, $V_{0}, k, \bar{a}, \eta$, $\phi$ were defined in Table 1 and $a=\frac{I D R_{d}}{k_{e} V_{0_{e}}}=9.38 \mathrm{mg} \mathrm{L}^{-1} \mathrm{mV}^{-1}$ [11].

\section{Conclusion}

This work quantitatively evaluated the performance of DMs-augmented MFC-assisted dye decolorization due to synergistic stimulation of electron-transport taking place in MFCs. Supplementation of DMs of textile dyes could simultaneously facilitate both rates of BG and DD. However, higher dye concentrations were more electrochemically favourable for DD than BG. Considering practical operation, whether DM-supplementation was synergistic or antagonistic to DD and BG strongly depended upon interactive characteristics of DMs and mother dye species with salts. With this scheme of assessment, batch or plug flow mode of operation with recycled streams to minimize reactor size as optimal reactor strategy of MFC-assisted WW decolorization could be considered for practicability.

\section{Acknowledgements}

The authors sincerely appreciate financial support (MOST 102-2221-E-197-016-MY3) from Taiwan's Ministry of Science and Technology for the project of $\mathrm{MFCs}^{\text {sdg. }}$. This study was completed as part of cooperative achievements for Academic Exchange Program between Yan-Tai University (China) and National I-Lan University (Taiwan) in 2015-2016 and as part of cooperative research association between NIU and Fushan Botanical Garden and Nature Centre, Taiwan Forestry Research Institute, Council of Agriculture, Executive Yuan, R.O.C. (Taiwan) (Article \# 1042600316, 2015/05/11).

\section{References}

[1] X. Z. Wang, X. Cheng, and D. Sun, "Autocatalysis in Reactive Black 5 biodecolorization by Rhodopseudomonas palustris W1," Appl Microbiol. Biotechnol., vol. 80, pp. 907-915, 2008.

[2] J. Sun, W. Li, Y. Li, Y. Hu, and Y. Zhang, "Redox mediator enhanced simultaneous decolorization of azo dye and bioelectricity generation in air-cathode microbial fuel cell," Bioresour. Technol., vol. 142, pp. 407-414, 2013.

[3] D. R. Bond, D. E. Holmes, L. M. Tender, and D. R. Lovley, "Electrode-reducing microorganisms that harvest energy from marine sediments," Science, vol. 295, pp. 483-485, 2002.

[4] B. Y. Chen, C. C. Hsueh, S. Q. Liu, I. S. Ng, and Y. M. Wang, "Deciphering mediating characteristics of decolorized intermediates for reductive decolorization and bioelectricity generation," Bioresour. Technol., vol. 145, pp. 321-325, 2013.

[5] B. Y. Chen, B. Xu, L. J. Qin, J. C. Lan, and C. C. Hsueh, "Exploring redox-mediating characteristics of textile dyebearing microbial fuel cells: thionine and malachite green," Bioresour. Technol., vol. 169, pp. 277-283, 2014.

[6] M. M. Zhang, W. M. Chen, B. Y. Chen, C. T. Chang, C. C. Hsueh, Y. Ding, K. L. Lin, and H. Xu, "Comparative study on characteristics of azo dye decolorization by indigenous decolorizers," Bioresour. Technol., vol. 101, pp. 2651-2656, 2010.

[7] B. Y. Chen, B. Xu, P. L. Yueh, K. Han, L. J. Qin, and C. C. Hsueh, "Deciphering electron-shuttling characteristics of thionine-based textile dyes in microbial fuel cells," J. Taiwan Inst. Chem. Engrs., vol. 51, pp. 63-70, 2015. 
[8] H. P. Luo, G. L. Liu, R. D. Zhang, and S. Jin, "Phenol degradation in microbial fuel cells," Chem. Eng. J., vol. 147, pp. 259-264, 2009.

[9] J. Sun, Y. Y. Hu, Z. Bi, and Y. Q. Cao, "Simultaneous decolorization of azo dye and bioelectricity generation using a microfiltration membrane air-cathode single-chamber microbial fuel cell," Bioresource Technol., vol. 100, pp. 31853192, 2009.

[10] I. Gajda, J. Greenman, C. Melhuish, and I. Ieropoulos, "Simultaneous electricity generation and microbially-assisted electrosynthesis in ceramic MFCs," Bioelectrochem, vol. 104, pp. 58-64, 2015.

[11] K. Han, P. L. Yueh, L. J. Qin, C. C. Hsueh, and B. Y. Chen, "Deciphering synergistic characteristics of microbial fuel cell-assisted dye decolorization," Bioresour. Technol., vol. 196, pp. 746-751, 2015.

[12] B. Y. Chen, M. M. Zhang, C. T. Chang, Y. Ding, K. L. Lin, C. S. Chiou, C. C. Hsueh, and H. Xu, "Assessment upon azo dye decolorization and bioelectricity generation by Proteus hauseri," Bioresour. Technol., vol. 101, pp. 4737-4741, 2010.

[13] O. Levenspiel, "Design for Parallel Reactions," in Chemical Reaction Engineering, 3rd Ed. New York: John Wiley\&Sons, 1999, pp. 152-169. 